Europhys. Lett., 61 (5), pp. 625-631 (2003)

\title{
Persistent patterns in deterministic mixing flows
}

\author{
A. Pikovsky and O. Popovych \\ Department of Physics, University of Potsdam \\ PF 601553, D-14415 Potsdam, Germany
}

(received 9 August 2002; accepted in final form 13 December 2002)

PACS. 47.52.+j - Fluid dynamics: Chaos.

PACS. 05.40.Jc - Brownian motion.

\begin{abstract}
We present a theoretical approach to the description of persistent passive scalar patterns observed in recent experiments with non-turbulent time-periodic two-dimensional fluid flows. The behaviour of the passive scalar is described in terms of eigenmodes of the evolution operator which coincides with the Frobenius-Perron operator for the corresponding Lagrangian dynamics with small noise; the latter represents the molecular diffusion. The asymptotic behaviour is dominated by the eigenmode with the slowest decay rate, which is shown to be localized in the non-mixing region of the flow.
\end{abstract}

Recently, in a number of experiments [1-3] it has been demonstrated that during chaotic advection in a fluid, a dye can form long-living, persistent structures. From the theoretical point of view, the problem is of asymptotic behaviour of solutions of linear equations for a passive scalar. Because of the linearity, the description in terms of eigenmodes is appropriate [4]. However, the determination of these modes for chaotically mixing flows is non-trivial (cf. a similar approach to the dynamo problem [5]).

In this letter we demonstrate that qualitative features of the persistent structures in chaotic fluid mixing observed in [1] can be modeled by flows having both chaotic and regular components (mixed phase space). Because there are non-mixing regions in the phase space, the long-time evolution toward the uniform concentration of the passive scalar density depends on the molecular diffusion constant. We study the spectrum of the eigenmodes for models of not globally chaotic flows and investigate the dependence of the decay rates of the passive scalar density on the parameters and the diffusion constant. It is worth noting that the same problem appears in the description of the correlations decay for chaos, the role of molecular diffusion being taken by additive noise; we extensively use this analogy below.

The evolution of a passive scalar density $\phi(x, y, t)$ in a two-dimensional incompressible flow $\boldsymbol{V}(x, y, t)$ is described by the equation

$$
\frac{\partial \phi}{\partial t}+\boldsymbol{V}(\boldsymbol{x}, t) \cdot \nabla \phi=D \Delta \phi,
$$

where $D$ is the molecular diffusion coefficient. Sometimes, a source term is included in the r.h.s. of eq. (1) in order to have statistically stationary patterns. We consider a source-free evolution of an initial distribution $\phi(x, y, 0)$, corresponding to experiments [1]. The velocity field of the advecting flow can be derived directly from the Navier-Stokes equation with timeperiodic force [6]. In this letter, in order to show that eq. (1) can demonstrate persistent patterns already for very simple velocity fields $\boldsymbol{V}(x, y, t)$, we consider a model flow introduced (C) EDP Sciences 


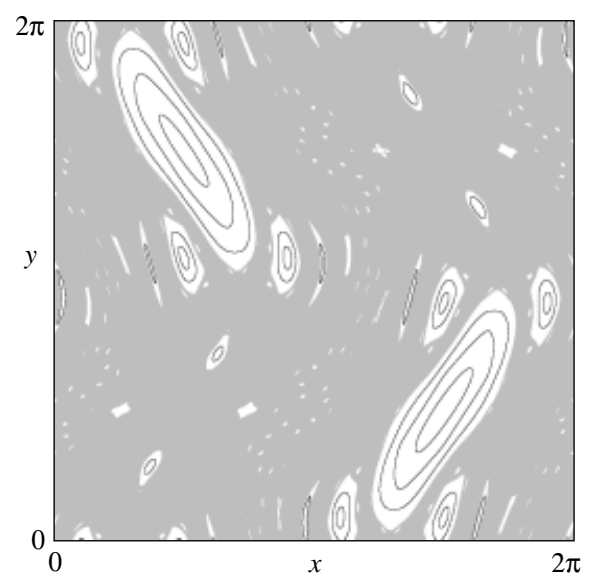

Fig. 1 - Phase portrait of map (4) for $L=2 \pi, T=1.0, U_{1}=2.6, U_{2}=5.48$ corresponding to a stroboscopic view (over the period $t=T$ ) of the trajectories of the flow (2). One long trajectory filling out a chaotic region is depicted in gray, it leaves elliptic islands blank: a few closed invariant curves with quasiperiodic dynamics are shown in black.

in $[4,7]$. This flow is periodic in $x$ and $y$ with period $L$ (i.e., it is in fact defined on a torus), and has the form

$$
\boldsymbol{V}(x, y, t)=\left(\begin{array}{l}
U_{1} f_{1}(t) \cos \frac{2 \pi y}{L} \\
U_{2} f_{2}(t) \cos \frac{2 \pi x}{L}
\end{array}\right) .
$$

We choose the functions $f_{1,2}$, following [7], as piecewise constant and time periodic with period $T$ :

$$
f_{1}(t)=1-f_{2}(t)= \begin{cases}1, & 0 \leq t<T / 2 \\ 0, & T / 2 \leq t<T\end{cases}
$$

This allows one to write the trajectories of particles in the flow explicitly [7]; the transformation of the particle coordinates after one period reads

$$
x_{n+1}=x_{n}+\frac{1}{2} U_{1} T \cos \left[\frac{2 \pi}{L} y_{n}\right], \quad y_{n+1}=y_{n}+\frac{1}{2} U_{2} T \cos \left[\frac{2 \pi}{L} x_{n+1}\right],
$$

where $n=t / T$ is the discrete time. This area-preserving two-dimensional mapping demonstrates a phase space typical of Hamiltonian flows with regular islands and chaotic sea, see fig. 1 .

In order to follow the evolution of the passive scalar field, we solved eq. (1) numerically, using the spectral method suggested in [7]. A system of complex ODEs for $201 \times 201$ first Fourier modes of the scalar $\phi$ has been numerically integrated. The results are presented in fig. 2. One can see that the evolution of the passive scalar field is qualitatively rather close to that observed in experiments [1] in the following sense: after a few periods of forcing, the passive scalar density (like the fluorescent dye in the experiments) forms a certain spatial structure which persists and only its contrast slowly decays with time toward the uniform state. Below, we focus on quantitative properties of these long-living structures.

For analysis, it is of advantage to have a discrete-in-time model of the passive scalar advection. This can be easily constructed from (1), if we split the operators of advection and 


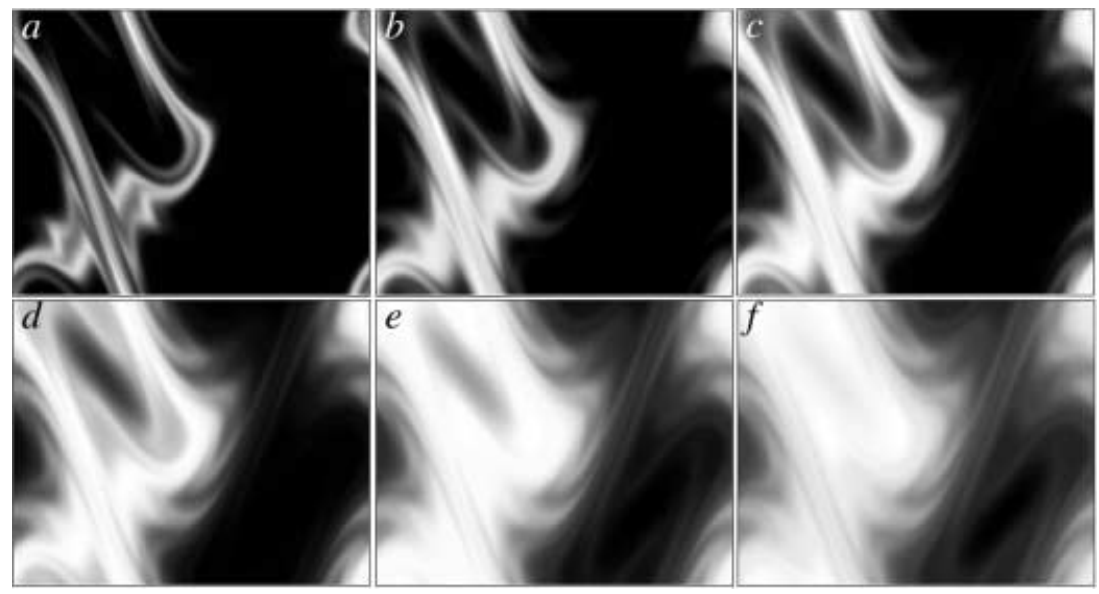

Fig. 2 - The field $\phi(x, y, t)$ (for the same parameters as in fig. 1 and $D=0.001$ ) is shown at times (a) $t=10 T$, (b) $t=20 T$, (c) $t=40 T,(d) t=80 T$, (e) $t=150 T$, and $(f) t=250 T$. The initial state $\phi(x, y, 0)$ is taken in the Gaussian form with mean $(1.6 ; 1.6)$ and standard deviation 0.15. The intensity of gray scaled from black to white corresponds to the variation of the magnitude of $\phi(x, y, t)$ from its minimum to its maximum.

diffusion. In the case of periodic in time velocity field $\boldsymbol{V}(x, y, t+T)=\boldsymbol{V}(x, y, t)$ the solution of the particle advection within the period can be written as a two-dimensional area-preserving mapping:

$$
x(n+1)=X(x(n), y(n)), \quad y(n+1)=Y(x(n), y(n)),
$$

where $n=t / T$ is the discrete time (e.g., (4) is a particular case of mapping (5) for the flow $(2,3))$. The density of the passive scalar evolving according to $(5)$ is governed by

$$
\phi(x, y, n+1)=\hat{P} \phi(x, y, n)=\iint \delta[x-X(u, v)] \delta[y-Y(u, v)] \phi(u, v, n) \mathrm{d} u \mathrm{~d} v .
$$

This equation describes the evolution of the density without diffusion. To take into account diffusion, we apply a discrete diffusion operator $\hat{Q}=\exp \left[D T \nabla^{2}\right]$ (which follows from the solution of (1) in the absence of advection) to obtain

$$
\begin{aligned}
\phi(x, y, n+1)= & \hat{Q} \hat{P} \phi(x, y, n)= \\
= & \frac{1}{4 \pi D T} \iint \mathrm{d} u \mathrm{~d} v \iint \mathrm{d} \xi \mathrm{d} \eta \exp \left[-\frac{(x-\xi)^{2}+(y-\eta)^{2}}{4 D T}\right] \times \\
& \times \delta[\xi-X(u, v)] \delta[\eta-Y(u, v)] \phi(u, v, n)= \\
= & \frac{1}{4 \pi D T} \iint \mathrm{d} u \mathrm{~d} v \exp \left[-\frac{(x-X(u, v))^{2}+(y-Y(u, v))^{2}}{4 D T}\right] \phi(u, v, n) .
\end{aligned}
$$

Equation (7) describes a model situation in which finite particle displacements occur instantaneously and these displacements are then followed by a period in which diffusion acts in the absence of advection. Note that eq. (7) allows us another physical interpretation. Namely, it describes the dynamics of the map (5) with additive noise:

$$
x(n+1)=X(x(n), y(n))+\sigma s_{1}, \quad y(n+1)=Y(x(n), y(n))+\sigma s_{2},
$$




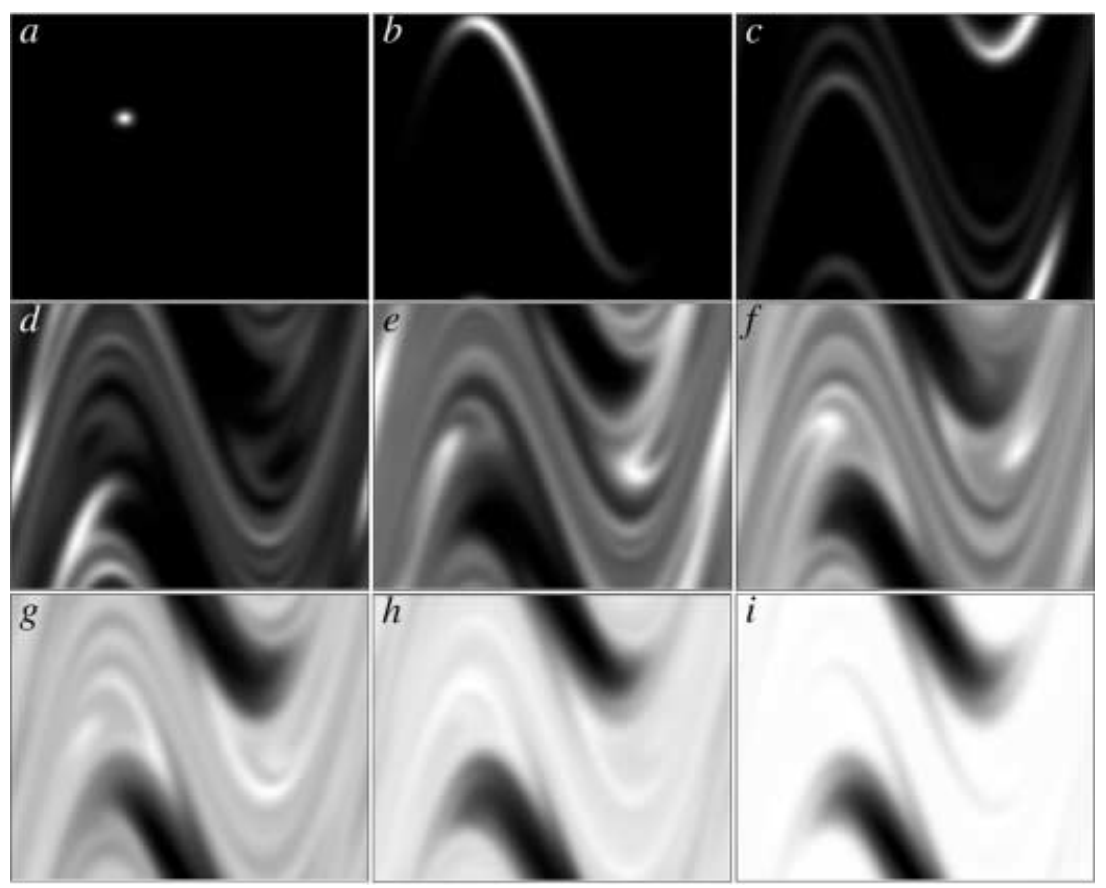

Fig. 3 - Relaxation toward the uniform invariant density for the noisy standard map (9) with $K=4$, $\sigma=0.1$, under the action of its Frobenius-Perron operator. An initial distribution $(a)$ taken in the Gaussian form, the first seven even iterations $(b)-(h)$, and the state after 150 iterations $(i)$ are shown. The intensity of gray scaled from black to white corresponds to the variation of the distribution density magnitude from its minimum to its maximum.

where $s_{1}, s_{2}$ are independent Gaussian random variables with zero mean and unit variance. The invariant probability density for this map obeys the Frobenius-Perron (FP) operator, which coincides with (7) if we choose $\sigma^{2}=2 D T$ (so we call hereafter the operator $\hat{Q} \hat{P}$ as FP operator). For the original advection problem, this means that we can model a smearing of the passive scalar density due to diffusion by adding random perturbations to deterministic trajectories, according to (8).

In the following we consider a particular case of the standard map dynamics, i.e., we write (5) as

$$
x(n+1)=x(n)+y(n) \quad(\bmod 2 \pi), \quad y(n+1)=y(n)+K \sin (x(n+1)) \quad(\bmod 2 \pi) .
$$

This map shares general properties of area-preserving maps to possess regions of regular and chaotic behaviour, and is very convenient for numerical analysis. Indeed, the FP operator (7) for the standard map has a simple form when written for Fourier harmonics of the density (cf. [8]):

$$
\psi_{n+1}(l, m)=\exp \left[-\left(l^{2}+m^{2}\right) D T\right] \sum_{j} \psi_{n}(j, m+j) J_{j-l}(m K),
$$

where $J_{j}(s)$ are Bessel functions and $\psi_{n}(l, m)=\frac{1}{4 \pi^{2}} \iint \mathrm{d} x \mathrm{~d} y \phi(x, y, n) e^{-i(l x+m y)}$.

A natural numerical approximation is to cut the Fourier series at some large number of harmonics (this number depends, of course, on $D T$ ) and to operate with finite matrices. In this 
TABLE I - Three maximal eigenvalues of the FP operator for the noisy standard map (9) for $K=4$.

\begin{tabular}{cccc}
\hline & $\sigma=0.06$ & $\sigma=0.10$ & $\sigma=0.14$ \\
\hline$\lambda_{0}$ & 1.0 & 1.0 & 1.0 \\
$\lambda_{1}$ & 0.9818 & 0.9626 & 0.9396 \\
$\lambda_{2}$ & 0.9160 & 0.8343 & 0.7378 \\
\hline
\end{tabular}

way an example of the evolution of initially localized density shown in fig. 3 was obtained. A long-living structure (fig. 3i) is formed after a short transient (fig. 3h) similarly to the evolution depicted in fig. 2 .

In order to characterize the long-time evolution of passive scalar structures we need to know the long-time asymptotic of the solutions of the FP equation (7). The latter is clearly determined by the spectral properties of the linear FP operator. It has an eigenvalue 1 corresponding to a uniform density, all other eigenvalues are less than 1 in absolute value. Thus, all spatially inhomogeneous modes decay, and for large times the dominant mode is that with the eigenvalue closest to one in absolute value. This is well confirmed for the example in fig. 3 when we compare the state at large time (see, e.g., fig. $3 i$ ) with the eigenmode of the FP operator corresponding to the second largest eigenvalue (fig. $4 a$ ). The eigenmode corresponding to the third largest eigenvalue $\lambda_{2}$ is presented in fig. $4 b$.

From this consideration it follows that asymptotically the contrast of the pattern decays exponentially, with the rate given by the second largest eigenvalue. Indeed, for large $n$ we can write the evolution of the passive scalar density as

$$
\phi(x, y, n)=\phi_{0}+\phi_{1}(x, y) \lambda_{1}^{n}
$$

where $\lambda_{1}$ is the eigenvalue closest to one and $\phi_{1}$ is the corresponding eigenmode. The contrast is defined as

$$
\delta \phi^{2}=\left\langle(\phi-\langle\phi\rangle)^{2}\right\rangle
$$

where the averaging means the integration over the spatial domain. Substituting here (11), we immediately get $\delta \phi(n) \propto \lambda_{1}^{n}$. The exponential asymptotic decay of the contrast is illustrated in fig. $5 a$. The decay rates with a good accuracy coincide with the eigenvalues of the FP operator presented in table I.

In the context of the map (8), to the decay of the contrast there corresponds the decay of correlations. For good (hyperbolic) chaotic sets this decay is exponential and is related to

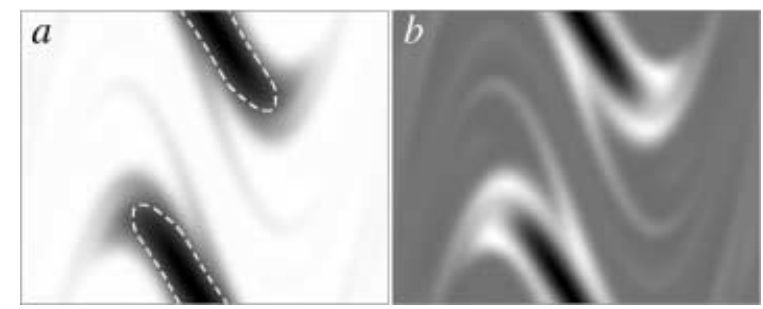

Fig. 4 - Eigendensities of the FP operator of the noisy standard map (same parameters as in fig. 3) corresponding to $(a)$ the second $\lambda_{1}$ and $(b)$ the third $\lambda_{2}$ maximal eigenvalues given in table I. Gray scaling from black to white corresponds to the variation of eigendensity magnitude from its minimum to maximum. The white dashed curve in $(a)$ outlines the biggest elliptic island of the noise-free standard map. 

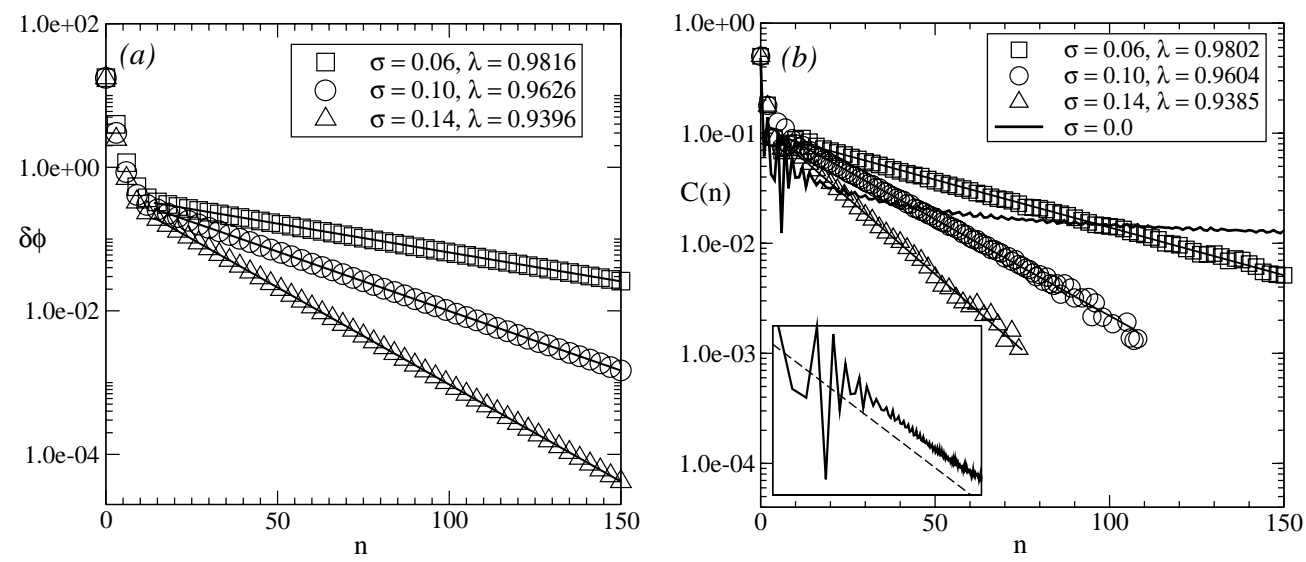

Fig. 5 - (a) Time evolution of the distribution density variance $\delta \phi$ under action of the FrobeniusPerron operator (10) for $K=4$. After a short transient, it decreases according to an exponential law $\delta \phi(n) \sim \lambda^{n}$, where the exponent $\lambda$ depends on the noise intensity $\sigma$. The presented values of $\lambda$ have been obtained by fitting the tails (solid lines). (b) The same analysis for the autocorrelation function $C(n)=\lim _{N \rightarrow \infty} \frac{1}{N} \sum_{m=0}^{N-1} g(m+n) g(m)$ of the observable $g(m)=\cos [x(m)+y(m)]$ for the noisy standard map (9). In both cases the empirically found decay rate agrees very well with the second eigenvalue of the FP operator (table I). A power law decay of correlations for the noise-free $(\sigma=0)$ standard map is shown in log-log scale in the subplot. The dashed straight line given for a comparison has the slope -0.5 .

the Ruelle-Policott resonances [9]. For systems with mixed phase space the situation is more complex (cf. $[8,10])$. The reason for this lies in the non-trivial picture of correlations decay which is asymptotically a power law. The power law decay of correlations is a phenomenon of universal nature for Hamiltonian dynamics and has thoroughly been investigated for different systems with discrete and continuous time (see, e.g., [11]). It is hardly feasible to follow the decay of contrast for the passive scalar dynamics without molecular diffusion, because any reduction of the transformation (10) to a finite number of modes means introduction of small diffusion. These features are easy to check for correlations in a noise-free map, calculating the correlation function from one long trajectory (see subplot in fig. $5 b$ ).

With finite noise the power law long-range correlations disappear, and an exponential decay (with the rate depending on the noise intensity) is observed as in hyperbolic chaotic systems. The correlations in (8) are presented in fig. $5 b$, which gives one more indication that the system is dominated by the second eigenvalues $\lambda_{1}$ of the FP operator given in table I and that their values do not depend on the representation (10).

It appears from table I that the second largest eigenvalue approaches 1 as the diffusion constant $D \sim \sigma^{2}$ tends to zero. A simple estimation based on the neglect of the advection terms in (1) gives $\lambda_{1} \sim 1-\sigma^{\alpha}$ with $\alpha=2$. However, in numerical estimations we could not confirm this power law, since we operate with finite matrices. Our best results with $501 \times 501$ Fourier modes in (10) gave $\alpha \approx 1.8$ with a slow tendency for this quantity to grow with decreasing $\sigma$. A reason for this may be in the non-trivial limiting case of vanishing diffusion. Indeed, one can expect that the noise-free FP operator has discrete and continuous spectra on the unit circle and inside it (cf. [12]). A small amount of noise destroys the continuous part of the spectrum [13], while discrete eigenvalues lying on the unit circle and being generally stable with respect to perturbations [14] start to move inside the unit disk (see also $[8,10]$ ) 
with the above power law dependency on the noise intensity.

In conclusion, we have demonstrated in this letter that qualitative features of persistent structures in chaotic fluid mixing can be modeled if we assume that the flow is not perfectly mixing, but has regular lacunae. In the latter the flow, although is stretching, does not mix, and certain structures localized at these places decay very weakly if the molecular diffusion $D$ is small. Formation and evolution of the persistent spatial structures can be modeled by means of discrete Hamiltonian maps with noise, where the noise corresponds to the molecular diffusion in the continuous system. The noise added to the chaotic dynamics ensures a destruction of regular islands in the chaotic sea. Then, the long-living structures are described by the eigenmodes of the Frobenius-Perron operator of the noisy map, and exactly the same modes are responsible for long correlations in the chaotic system. The corresponding eigenvalues of the FP operator determine the decay rates of correlations and contrast of patterns. We have demonstrated that regular islands are important for the relevant modes, contrary to the case of magnetic dynamo [5], where the relevant modes are located in the most chaotic regions. In order to simplify the consideration, in this letter we deal with an illustrative model system, the standard map, and present only one example of persistent spatial structure located in the elliptic island. The extended description of several other types of structures that we have found for more fluid-relevant systems will be published elsewhere. We believe that the same program of investigation can be accomplished for realistic equations describing experimental flow patterns.

We thank B. ECKHARDT, R. Livi and T. TÉL for many fruitful discussions. OP acknowledges financial support from the Alexander-von-Humboldt Foundation.

\section{REFERENCES}

[1] Rothstein D., Henry E. and Gollub J. P., Nature, 401 (1999) 770.

[2] Deval J. and Tabeling P., Phys. World, January, 13 (2000) 22.

[3] Voth G. A., Haller G. and Gollub J. P., Phys. Rev. Lett., 88 (2002) 254501.

[4] Pierrehumbert R. T., Chaos, Solitons Fractals, 4 (1994) 1091.

[5] Childress S. and Gilbert A. D., Stretch, Twist, Fold: The Fast Dynamo (Springer-Verlag, Berlin, Heidelberg) 1995.

[6] Eckhardt B. and Hascö̈t E., private communications.

[7] Antonsen T. M., Fan Z., Отt E. and Garcia-Lopes E., Phys. Fluids, 8 (1996) 3094.

[8] Khodas M. and Fishman S., Phys. Rev. Lett., 84 (2000) 2837; Phys. Rev. E, 62 (2000) 4769.

[9] Ruelle D., Phys. Rev. Lett., 56 (1986) 405.

[10] Weber J., Hanke F. and Šeba P., Phys. Rev. Lett., 85 (2000) 3620; Weber J., Haake F., Braun P. A., Manderfeld C. and Šeba P., J. Phys. A, 34 (2001) 7195.

[11] Chirikov B. V. and Shepelyansky D. L., Physica D, 13 (1984) 395; Chirikov B. V. and Shepelyansky D. L., Phys. Rev. Lett., 82 (1999) 528; Meiss J. D. and Отt E., Physica D, 20 (1986) 387; Zaslavsky G. M. and Niyazov B. A., Phys. Rep., 283 (1997) 73; Artuso R., Physica D, 131 (1999) 68; Yuri M., Nonlinearity, 15 (2002) 429; Geisel T., Zacherl A. and Radons A., Phys. Rev. Lett., 59 (1987) 2503; Floriani E. and Lima R., Chaos, 9 (1999) 715.

[12] Arnold V. I. and Avez A., Ergodic Problems of Classical Mechanics (Addison-Wesley, New York) 1989.

[13] Huisinga W., The Essential Spectral Radius and Asymptotic Properties of Transfer Operators, submitted to Dyn. Syst. Appl., preprint available from http://www.math.fu-berlin.de/ rhuisinga/.

[14] Blank M. and Keller G., Nonlinearity, 11 (1998) 1351. 